Revue internationale P.M.E.

Économie et gestion de la petite et moyenne entreprise

\title{
Processus de désagrégation du système des grandes entreprises nationales en Hongrie
}

\section{Györgyi Barta et Erzsébet Poszmik}

Volume 5, numéro 1, 1992

URI : https://id.erudit.org/iderudit/1008133ar

DOI : https://doi.org/10.7202/1008133ar

Aller au sommaire du numéro

Éditeur(s)

Presses de l’Université du Québec

ISSN

0776-5436 (imprimé)

1918-9699 (numérique)

Découvrir la revue

Citer cet article

Barta, G. \& Poszmik, E. (1992). Processus de désagrégation du système des grandes entreprises nationales en Hongrie. Revue internationale P.M.E., 5(1), 63-75. https://doi.org/10.7202/1008133ar

\section{Résumé de l'article}

Le résultat le plus spectaculaire concernant le changement actuel du système économique en Hongrie est la transformation radicale du système d'organisations industrielles. La variété des nouveaux organismes économiques démontre une véritable transition du secteur privé, auparavant insignifiant et limité, vers une économie fonctionnant sur un mode plus ou moins privé.

Cette analyse montre qu'entre 1985 et 1989, le nombre des entreprises a pratiquement doublé. Après 1989, un laps de temps d'une année fut suffisant pour que le nombre d'entreprises double à nouveau. Cette augmentation est due surtout à l'apparition de PME entre 1985 et 1989 mais aussi, par la suite, à la division des grandes et des moyennes entreprises.

Les auteurs présentent les principaux facteurs ayant favorisé la désagrégation des grandes entreprises en décrivant la diminution du rôle de celles-ci et la situation instable des dirigeants des grandes entreprises dans une période de changement de système politique. Enfin, des considérations sur le succès de ce changement structurel sont présentées selon les différentes formes qu'ont prises les divisions d'entreprises ainsi que les diverses raisons qui ont amené les entreprises à ce changement.
Ce document est protégé par la loi sur le droit d'auteur. L'utilisation des services d'Érudit (y compris la reproduction) est assujettie à sa politique d'utilisation que vous pouvez consulter en ligne.

https://apropos.erudit.org/fr/usagers/politique-dutilisation/ 


\title{
Processus de désagrégation du système des grandes entreprises nationales en Hongrie
}

\author{
Györgyi BARTA \\ Académie des sciences de Hongrie* \\ Erzsébet POSZMIK \\ Université d'économie de Budapest"*
}

\begin{abstract}
RÉSUMÉ
Le résultat le plus spectaculaire concernant le changement actuel du système économique en Hongrie est la transformation radicale du système d'organisations industrielles. La variété des nouveaux organismes économiques démontre une véritable transition du secteur privé, auparavant insignifiant et limité, vers une économie fonctionnant sur un mode plus ou moins privé.
\end{abstract}

Cette analyse montre qu'entre 1985 et 1989, le nombre des entreprises a pratiquement doublé. Après 1989, un laps de temps d'une année fut suffisant pour que le nombre d'entreprises double à nouveau. Cette augmentation est due surtout à l'apparition de PME entre 1985 et 1989 mais aussi, par la suite, à la division des grandes et des moyennes entreprises.

Les auteurs présentent les principaux facteurs ayant favorisé la désagrégation des grandes entreprises en décrivant la diminution du rôle de celles-ci et la situation instable des dirigeants des grandes entreprises dans une période de changement de système politique. Enfin, des considérations sur le succès de ce changement structurel sont présentées selon les différentes formes qu'ont prises les divisions d'entreprises ainsi que les diverses raisons qui ont amené les entreprises à ce changement.

\section{ABSTRACT}

The most spectacular result concerning present changes in the Hungarian economic system is the radical transformation of industrial organizations. The variety of new economic organisms illustrates a true transition of the private sector, before insignificant and limited, toward a functionning economy of a more or less private style.

* Györgyi Barta, économiste, chercheur au Centre régional de recherche de l'Académie des sciences de Hongrie.

** Erzsébet Poszmik, économiste, assistant à la chaire de micro-économie, Université d'économie de Budapest. 
This analysis shows that between 1985 and 1989 the number of enterprises almost doubled again. After 1989, in only one year, the number of enterprises doubled. This increase occured largely because of the emergence of SMBS between 1985 and 1989 but also, afterwards, by the division of large and middle-sized businesses.

The authors present the principal factors which caused the disintegration of large businesses; their diminishing role and the unstable situation of largebusiness leaders at a time when the political system was changing. Finally, the success of this structural change is considered from the point of view of the different forms these divisions took as well as the various reasons that lead the businesses to change.

\section{RESUMEN}

El resultado mas espectacular concerniente al cambio actual del sistema económico en Hungría es la transformacion radical del sistema de las organizaciónes industriales. La variedad de nuevos organismos económicos demuestran una verdadera transicion del sector privado, anteriomente insignificante y limitado, hacia una economia que funciona de une manera mas o menos privada.

Este estudio muestra que entre 1985 y 1989 el número de empresas seha duplicado praticamente. Despues de 1989, un año hasido suficiente para que el número de empresas se duplique de nuovo. Esta augmentacion es debida sobretodo a la aparicion de PME entre 1985 y 1989 pero tambien, seguidamente, a la division de las grandes y de las medianas empresas.

Los autores presentan los principales factores que han favorecido el desagregamiento de las grandes empresas describiendo la diminucion del rol de las mismas y la situacion inestable de los dirigentes de las grandes empresas dentro un periodo de cambio del sistema político. Enfin, las consideraciónes sobre las aventajas de éste cambio estructural son presentadas segun las diferentes formas que han tomado las divisiones de las empresas como las diversas razones que han orientado a las empresas a éste cambio.

\section{Introduction}

Le résultat sans doute le plus spectaculaire du changement actuel de système économique en Hongrie est la transformation radicale en cours du système d'organisations industrielles. La variété des nouveaux organismes économiques démontre une véritable transition du secteur privé, auparavant insignifiant et limité dans son activité sous le règne du communisme (alors que même les coopératives industrielles de l'époque étaient plus proches du secteur public que des coopératives réelles), vers une économie fonctionnant sur un mode plus ou moins privé, avec toutes sortes d'organisations au détriment du secteur public. 
Mais cette tendance actuelle, dirigée ou spontanée, vers de nouveaux rapports de propriété ne suffit pas pour changer le système. Le nouvel environnement économique, plus difficile et fondamentalement différent, exige une transformation des organisations. L'apparition de nouvelles formes d'organisations - même sans changement des rapports de propriété - permet d'espérer une meilleure adaptation des firmes aux nouvelles conditions économiques. Ainsi, les entreprises doivent accepter les nouvelles règles de rentabilité : quelle activité doit-elle soutenir ? de quelle autre doit-elle se défaire ? Les expériences actuelles montrent que les grandes entreprises divisent leurs activités en unités partiellement indépendantes, à l'encontre de la centralisation verticale antérieure. Aussi, de plus en plus d'unités ou même d'entreprises doivent d'être liquidées. Tout ceci est complété par une augmentation folle du nombre des petites entreprises. Finalement, nous assistons à une très forte multiplication des organisations économiques, un retour à une taille plus normale des entreprises et à une structuration caractéristique des pays développés capitalistes.

Dans l'industrie manufacturière, cette restructuration ne date pas de l'automne 1989, ni du printemps 1990, bien qu'elle se soit accélérée après le changement « officiel » du système. La première intervention importante sur la structure industrielle de l'organisme, jusque-là inchangée malgré la réforme de 1968, eut lieu en 1982. C'est à ce moment que furent légalisées les sociétés économiques à caractère privé dites collectivités économiques de travail. Ceci favorisa l'expansion des activités salariées, avec pour résultat l'augmentation des revenus personnels plutôt que le démarrage d'entreprises indépendantes. De plus, les conditions pour assurer la concurrence n'étaient pas les mêmes pour les petites ou les grandes entreprises selon les différents secteurs. Toutefois, plusieurs petites entreprises du secteur privé ont pu arriver au succès malgré des conditions défavorables.

La deuxième étape importante fut la préparation et le vote de la loi sur les sociétés en 1988. Cette loi sur les sociétés réglementait les créations de nouvelles sociétés dans le cadre d'un modèle théorique d'économie de marché communiste. Elle rendait possible, à côté des entreprises nationales, publiques, d'autres formes d'organisation à propriété collective nationale ainsi que des entreprises de type associatif. Elle encourageait la création d'entreprises mixtes à l'aide de capitaux étrangers. Tout ceci signifiait la construction d'une économie mixte s'appuyant en priorité sur le secteur public. La loi sur les sociétés rendit possible qu'une partie de l'entreprise (un atelier), ou une de ses activités (par exemple, le développement d'une technologie), se transforme en société indépendante. Ainsi, sous l'effet de cette loi, la décentralisation des grandes entreprises s'accéléra. La plupart de ces sociétés restèrent cependant propriété de la société-mère. Mais il existe aussi des exemples de formation de sociétés indépendantes (partenaires des coopératives ou des banques, etc.). Ces nouvelles 
sociétés préparèrent la période de privatisation accélérée de 1990-1991. Mais pour cela, une autre loi importante fut également nécessaire : la loi dite de « conversion ». Celle-ci entra en vigueur en 1989. Elle réglemente maintenant la conversion des entreprises nationales en sociétés à responsabilité limitée ou sociétés anonymes.

Ce type de conversion est indispensable, puisqu'il assure l'apparition de véritables propriétaires. La privatisation hongroise est donc complétée par une transformation radicale de l'organisme et au cours de cette transformation, les entreprises nationales sont remplacées par des sociétés privées.

\section{Le changement de structure des entreprises manufacturières}

\section{TABLEAU 1 \\ Évolution du nombre d'entreprises et d'établissements manufacturiers en Hongrie entre 1980 et 1990}

\begin{tabular}{|c|c|c|c|}
\hline Année & $\begin{array}{c}\text { Nombre } \\
\text { d'entreprises }\end{array}$ & $\begin{array}{c}\text { Nombre } \\
\text { d'établissements }\end{array}$ & $\begin{array}{c}\text { Nombre } \\
\text { d'établissements } \\
\text { moyens } \\
\text { par entreprise }\end{array}$ \\
\hline 1980 & 1360 & 8997 & 6,6 \\
1982 & 1359 & 8551 & 6,3 \\
1984 & 1317 & 7775 & 5,9 \\
1985 & 1871 & 7737 & 4,1 \\
1987 & 2435 & 8806 & 3,6 \\
1989 & 3661 & 9886 & 2,7 \\
1990 & 6997 & 13336 & 1,9 \\
\hline
\end{tabular}

Source : Statistiques industrielles annuelles 1980-1990. Données de base des établissements manufacturiers 1990. 
Entre 1985 et 1989 , le nombre des entreprises a pratiquement doublé, renversant ainsi la tendance à la diminution enregistrée dans les années précédentes. Cette augmentation est due avant tout à l'apparition des PME. Après 1989, un laps de temps d'une année fut suffisant pour que le nombre des entreprises double. Pour ces deux dernières années, l'augmentation s'explique non seulement par la création de nouvelles entreprises, mais aussi par la division des grandes et moyennes entreprises (tableau 1).

TABLEAU 2

Répartition des entreprises manufacturières et des salariés selon le nombre d'établissements par entreprise

\begin{tabular}{|l|c|c|c|r|r|r|r|l|}
\hline $\begin{array}{c}\text { Nombre } \\
\text { d'établisse- } \\
\text { ments } \\
\text { par entreprise }\end{array}$ & \multicolumn{6}{|c|}{$\begin{array}{c}\text { Répartition du nombre } \\
\text { d'entreprises en \% }\end{array}$} & \multicolumn{5}{c|}{$\begin{array}{c}\text { Répartition du nombre } \\
\text { de salariés en \% }\end{array}$} \\
\hline & 1972 & 1982 & 1986 & 1990 & 1972 & 1982 & 1986 & 1990 \\
$1-3$ & 33,6 & 31,1 & 68,4 & 90,4 & 23,9 & 22,0 & 33,5 & 50,0 \\
$4-10$ & 38,7 & 39,3 & 21,1 & 7,2 & 39,7 & 36,5 & 34,5 & 29,0 \\
11 et + & 27,7 & 29,6 & 10,5 & 2,4 & 36,4 & 41,5 & 32,0 & 19,0 \\
\hline Total & 100,0 & 100,0 & 100,0 & 100,0 & 100,0 & 100,0 & 100,0 & 100,0 \\
\hline
\end{tabular}

Source : Données de base des établissements manufacturiers 1972, 1982, 1986 et 1990.

La forme de plusieurs établissements des entreprises qui caractérisait jusqu'alors l'industrie hongroise changea radicalement à partir du milieu des années 1980. Alors qu'en 1982, $70 \%$ des entreprises industrielles avaient au moins 4 établissements (représentant $78 \%$ des salariés), en 1990, la proportion des entreprises ayant plus de 4 établissements n'atteint plus $10 \%(50 \%$ des travailleurs). En 1990, la structure industrielle basée sur plusieurs établissements n'est plus une caractéristique du système industriel. Il est cependant incontestable que la concentration d'employés demeure toujours élevée $(9,6 \%$ des entreprises emploient 49,4\% des employés (tableau 2). 
TABLEAU 3

Variation de la répartition des entreprises manufacturières selon leur taille

\begin{tabular}{|l|l|r|r|r|}
\hline \multicolumn{2}{|c|}{ Catégories d'entreprises selon la taille } & 1982 & 1986 & \multicolumn{1}{|c|}{1990} \\
\hline moins de 20 & & 0,5 & 8,5 & 48,8 \\
$21-50$ & & 0,8 & 10,2 & 16,2 \\
$51-300$ & Petites & 37,6 & 42,6 & 21,8 \\
$301-1000$ & Moyennes & 33,5 & 21,9 & 8,8 \\
$1001-5000$ I & Grandes & 26,2 & 15,3 & 4,1 \\
$5001-10000$ II & Entreprises & 2,4 & 0,9 & 0,2 \\
10001 et plus III & & 1,0 & 0,6 & 0,1 \\
\hline & & 100,0 & 100,0 & 100,0 \\
\hline
\end{tabular}

Source : Données de base des établissements manufacturiers 1982, 1986 et 1990.

GRAPHIQUE 1

Changements de la taille des entreprises manufacturières en Hongrie

Catégorie d'entreprise

1982

\section{nombre d'employés}

1990

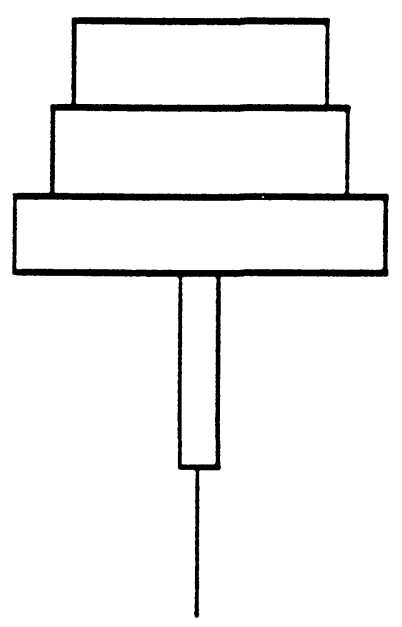

$1000<$

$301-1000$

$51 \cdot 300$

$21-50$

$<20$ 
La pyramide des entreprises selon la taille s'est donc retournée en un temps très court, comme on peut le voir au tableau 3 et au graphique 1 . Bien sûr, comme nous l'avons observé au niveau des entreprises avec plusieurs établissements, la concentration des employés a diminué dans une moindre mesure. Par exemple, en 1982, $29 \%$ des travailleurs manufacturiers étaient employés dans des entreprises de 5000 personnes; aujourd'hui, 13,5\% seulement le sont.

TABLEAU 4

Variation du nombre des grandes entreprises entre 1982 et 1990

\begin{tabular}{|l|r|r|r|}
\hline Catégories d'entreprises & 1982 & 1986 & 1990 \\
\hline $1000-5000$ & 326 & 320 & 287 \\
$5001-10000$ & 33 & 18 & 18 \\
$10000-$ & 14 & 13 & 4 \\
\hline
\end{tabular}

Source : Données de base des établissements manufacturiers, 1982, 1986 et 1990.

La définition des catégories d'entreprises selon le nombre d'employés est toujours arbitraire. Nous utilisons la pratique internationale selon laquelle les petites entreprises ont moins de 50 employés, les moyennes entreprises, de 50 à 1000 personnes et les grandes, plus de 1000 travailleurs. Nous avons aussi divisé la catégorie grande entreprise en trois sous-groupes, soit 1000 à 5000 , 5000 à 10000 et plus de 10000 employés, puisque cette division quantitative entraîne des variations qualitatives.

\section{Facteurs ayant favorisé la désagrégation des grandes entreprises}

\subsection{Les vingt dernières années de la pérlode Kádár (1968-1988)}

En 1968, l'introduction d'un nouveau mécanisme économique et la centralisation qui l'a directement précédé donnèrent aux grandes entreprises une position de pouvoir important. Pendant les vingt années suivantes de la période Kádár, la bureaucratie du Parti communiste et de l'État ainsi que les dirigeants des grandes entreprises devinrent les détenteurs du pouvoir. Ils se rendirent indispensables les uns aux autres ; le Parti communiste et la direction gouvernementale assura par des moyens politiques le pouvoir des chefs d'entreprises, 
alors que les grandes entreprises renforçaient le pouvoir politique par leur production économique. Ils devinrent partenaires sur le marché économique et politique. Dans leurs échanges, les dirigeants politiques firent appel au besoin d'une situation économique équilibrée pour limiter les exigences des grandes entreprises ; pour leur part, les dirigeants des grandes entreprises, lorsqu'ils voulurent augmenter leurs droits et exigences, s'appuyèrent sur leur rôle dans la satisfaction des besoins de la population, sur leur action dans le commerce international et sur les masses ouvrières travaillant dans leurs entreprises. C'est pourquoi en 1972, 50 très grandes entreprises se virent dispensées du système général de réglementation économique. De même, la pression des grandes entreprises donna lieu à une nouvelle vague de centralisation au début des années 70. Par les fusions et les assimilations, les grandes entreprises profitèrent de capitaux gratuits et d'une force de travail formée et expérimentée. Cependant, pour les grandes entreprises, l'avantage le plus important fut leur position acquise lors de la redistribution qui se traduisit par des crédits et des allocations préférentielles. À la fin des années 70 pourtant, la détérioration de la situation économique et l'augmentation au double de la dette extérieure devinrent évidentes. Au début, l'aide aux secteurs à mauvais rendement des grandes entreprises ne put se faire qu'au détriment d'autres entreprises et activités. Dans une période d'expansion générale, cette aide était possible, puisque les entreprises plus rentables que la moyenne gardaient un profit malgré les prélèvements pour aider les plus faibles. Plus tard, l'aide aux grandes entreprises ne fut possible qu'à partir de crédits extérieurs (étrangers). L'endettement général du pays favorisa le développement de la crise de la période Kádár. Le consensus entre les grandes entreprises et le Parti communiste chavira : le gouvernement et le parti essayèrent de limiter les dégâts par la décentralisation, la limitation des grandes entreprises, la légalisation des petites entreprises et le développement du secteur privé. Cependant, les grandes entreprises continuèrent à croître et à rechercher des crédits de développement.

Les efforts du gouvernement eurent un succès partiel. Jusqu'à la fin de 1985 , le nombre d'entreprises augmenta environ de 400, grâce à la décentralisation. La réorganisation dans les faits ne changea pas la structure État-grandes entreprises de l'industrie hongroise ; surtout elle ne permit pas l'amélioration de l'efficacité. Le processus de décentralisation tourna court dans la deuxième partie des années 80 .

Dans cette deuxième phase économique, les petites entreprises se développèrent avec succès et ainsi aidèrent au prolongement de la première période de crise économique. L'élargissement et le succès de ce secteur des PME furent cependant sévèrement limités ; dans le cadre du système socialiste, les possibilités de développement étaient faibles. Dans les années 80 , la crise s'approfondit ; le Parti communiste, la bureaucratie nationale et les chefs des grandes 
entreprises ne pouvaient pas, seuls, apporter le renouveau à l'intérieur du système économicosocial.

\subsection{La diminution du rôle des grandes entreprises, une situation instable des dirigeants des grandes entreprises dans une période de changement de systeme politique}

Il est difficile de tirer des conclusions sûres à partir des tendances des deux dernières années. Non seulement on assiste à des changements récents aux tendances incertaines (dont l'analyse scientifique ne fait que commencer), mais aussi, entre les dirigeants des grandes entreprises et la nouvelle direction politique, on assiste à une lutte de pouvoir qui n'est pas près de finir.

Ces deux dernières années sont caractérisées par des positions tendues, des coups fourrés et des marchandages secrets. Malgré tout, on peut penser que la position de pouvoir des grandes entreprises est ébranlée ; ces dernières se différencient par leur faculté d'adaptation aux circonstances : quelques-unes ont des chances de survivre au prix de gros efforts, d'autres végètent, d'autres encore périclitent.

Les caractéristiques des relations actuelles entre la bureaucratie du nouvel État et les grandes entreprises sont différentes de la situation précédente. La direction de l'industrie et des entreprises n'est plus responsables de l'approvisionnement, de l'obligation d'exporter à n'importe quel prix, de l'embauche de ses employés. Par contre, les entreprises ne peuvent plus compter sur la défense de leurs intérêts par l'État, sur ses crédits et ses allocations et sur une aide extérieure pour régler leurs problèmes de marché et d'introduction de nouveaux produits. Dans cette nouvelle situation pour les chefs d'entreprises, non seulement les partenaires habituels du marchandage (bureaucratie, Parti communiste) ont-ils disparu, mais est disparu avec eux le sens même du marchandage.

Les nouveaux partenaires des grandes entreprises sont l'Agence du Trésor National et les banques. Leurs relations sont maintenant de nature différente. Une relation compliquée entre les banques et la direction des grandes entreprises s'est installée, parce que, d'une part, dans beaucoup de cas les banques sont devenues actionnaires des mêmes entreprises, et que d'autre part, les banques commerciales gardent les entreprises sous leur contrôle financier. Aussi, ces dernières, dépendantes de la disponibilité des crédits accordés par les banques en fonction de leur productivité, se retrouvent dans une relation de dépendance mutuelle. À long terme cette relation devra renforcer la situation dominante des banques sur les entreprises ; autrement, les banques feraient vite faillite, si (comme durant la période du régime communiste) elles permettaient des crédits sans limites aux grandes entreprises en difficulté. Plus encore, la diminution 
implacable des possibilités de vente avec l'effondrement du marché soviétique et des autres pays communistes, ajoutée à la diminution de la consommation nationale, donna un coup fatal aux grandes entreprises.

Par exemple, la production des grandes structures industrielles nationales coopératives a diminué entre 1988 et 1989 de $9,4 \%$, de 8 à $9 \%$ au premier semestre de 1991 et au cours du second semestre de cette année-là, de 10 à $12 \%$. Cette diminution de production est la plus grande enregistrée depuis la Seconde Guerre mondiale. Il ne s'agit pas d'une redistribution, mais d'une diminution dans la plupart des secteurs industriels. La diminution de la production de $40 \%$ entre 1988 et 1990 dans l'industrie chimique et mécanique et de 20 à $25 \%$ dans l'industrie légère et minière est due en grande partie à la diminution des exportations vers l'Union soviétique. Mais la chaîne de coopération entre les grandes entreprises s'est également brisée avec le non-écoulement des produits fabriqués en grande quantité. La liste des entreprises en faillite, dont les dettes mises en recouvrement causèrent en 1990 des pertes de près de 300 milliards de Forints, s'allonge de jour en jour. Il est impossible de savoir, entre les dettes ou les demandes des entreprises figurant sur cette liste, lesquelles sont les plus grandes?

L'industrie manufacturière se trouve donc dans une situation de repli. Il serait malveillant d'affirmer que ceci résulte uniquement de la politique économique du gouvernement ; mais si l'effondrement du marché de l'Est a favorisé l'atteinte des nouveaux buts de la politique économique, en même temps, il a retiré le pouvoir des mains des dirigeants des grandes entreprises, pouvoir hérité de l'ancien régime politique.

Enfin, quant au dernier atout des grandes entreprises, l'emploi, on constate qu'entre 1988 et 1990 , le nombre des travailleurs de l'industrie a diminué de 190 000. En 1991, s'ajoutèrent 170000 licenciements, soit le tiers de l'effectif industriel de l'année 1988. À la suite de ces pertes d'emploi massives, il n'y a jusqu'à présent pas eu de manifestation de la classe ouvrière ; ceci s'explique par le fait qu'il n'existe pas, en réalité, de classe ouvrière unifiée. À l'intérieur même de cette classe, il existe de nombreux conflits d'intérêts réels ou imaginaires et on ne peut présager de l'identité des groupes de cette classe qui seront gagnants dans une économie de marché. Les intérêts divergents d'une classe ouvrière divisée ne peuvent être défendus par aucun parti ou syndicat.

Aussi sur les grandes entreprises, pèse une force extérieure venant de deux directions. La direction politique et économicopolitique ne veut plus discuter avec les dirigeants des grandes entreprises, ni aider par des moyens extérieurs celles qui ne peuvent s'adapter aux circonstances. De plus, la situation économique devenue très défavorable rend difficile pour la plupart des grandes entreprises la légitimation de leur existence par leurs performances. 


\subsection{La transformation de la structure et les possibilités de survie des grandes entreprises grâce à leurs ressources internes}

\section{Les formes ou le processus de transformation de la structure}

On peut trouver plusieurs prémisses non négligeables de la décentralisation actuelle et spectaculaire des organisations dans les années 80 . La direction des industriels et les entreprises elles-mêmes, pour faire face aux problèmes économiques de plus en plus graves, eurent souvent recours à une transformation

structurelle. À cette époque, la grande partie des grandes entreprises se libéra des cadres des grandes régies pour gagner plus d'indépendance. À l'intérieur même des entreprises, la décentralisation par la création d'unités comptables indépendantes (sous forme de centre de profits ou de frais), ou par la formation de divisions, a eu pour but de lier les succès ou les échecs de production aux secteurs intéressés ; ainsi, à la place d'une direction centrale dirigeant tout, on créa des directions indépendantes à pouvoirs de décision. Il faut remarquer que dans les entreprises où la décentralisation fut menée avec succès, la création ultérieure de société privée indépendante fut également plus efficace. Cependant, on poussa souvent le processus de démembrement là où l'interdépendance organisationnelle de la production ne le permettait pas. Dans ce cas, les sociétés non viables firent faillite.

Le vote des lois pour la création de sociétés privées et de transformations structurelles de 1988-1989 a représenté un tournant décisif dans la réorganisation des entreprises. La transformation des entreprises en sociétés économiques privées a aussi souvent entraîné un changement de directeur.

Comme les entreprises nationales sont obligées de se réorganiser en sociétés avant la fin de l'année 1992, il est évident que ce processus est général même si ces entreprises se trouvent à des stades très différents face à cette transformation. Comme la transformation de structure est la condition de la privatisation, le processus ne peut arriver à terme avant que les rapports de propriété ne se soient éclaircis.

Les grandes entreprises ont choisi des formes d'organisation très différentes. La forme la plus fréquente est la société anonyme. Souvent, elle se transforme en organisme de type holding dans lequel on peut trouver la société anonyme, la société à responsabilité limitée, la filiale ou la société mixte. Le but est de diviser les grandes entreprises en unités indépendantes bien séparées qui pourront être plus tard privatisées indépendamment les unes des autres. 


\section{Le succès dans ce changement structurel}

L'un des buts du changement structurel est d'évaluer le capital ou la valeur exacte de l'entreprise et sa rentabilité ou encore son succès éventuel sur le marché. Les divisions sous forme de sociétés peuvent se ranger dans plusieurs groupes selon diverses raisons d'entreprises. Souvent les unités performantes fonctionnant bien ont la responsabilité de se développer indépendamment d'une société mère aux prises avec plusieurs difficultés. Dans ce cas, en général, l'unité elle-même prend l'initiative de son indépendance, se libérant ainsi d'une société qui gruge ses profits. Dans d'autres cas, l'investisseur ne retient qu'une partie d'une société, le tout ne l'intéressant pas. Parfois, c'est la société qui essaie de se débarrasser d'une branche non rentable en la transformant en société indépendante. Ainsi, en comptant sur l'effet positif de cette libération, elle pense peutêtre permettre à cette branche de survivre. Au cours de la transformation, on a un surplus de main-d'œuvre et la composition de la force de travail devient inadéquate. Pratiquement toute société doit réduire ses effectifs lors de sa privatisation ; elle y est également obligée par le ralentissement économique. Souvent, lors de la création d'une nouvelle société, la modernisation des équipements se révèle indispensable pour assurer une meilleure rentabilité. Il est plus rentable de se libérer des stocks inutilisés et des outils périmés que de les reprendre dans la nouvelle société.

Le but final de la nouvelle organisation des entreprises publiques est leur privatisation, le changement des rapports de propriété et l'incorporation de capitaux étrangers. Sur ce plan, en Hongrie, les résultats sont encore modestes. La restructuration des entreprises prospères avec de bonnes possibilités de marché entraîne beaucoup de succès pour l'entreprise. L'apport de capitaux étrangers a aussi permis d'améliorer la rentabilité de plusieurs d'entre elles. Mais cela n'a pas touché les entreprises en difficulté et leur situation est demeurée fondamentalement précaire, malgré la décentralisation ou la transformation structurelle. Toutefois certains petits avantages apparaissent, même dans la situation difficile actuelle. Les chances de survie de ces entreprises ont augmenté et seront d'autant plus grandes dans la mesure où les conditions économiques deviendront plus favorables. Leur situation sur les marchés peut s'améliorer par la diversification, l'apport de capitaux et une direction plus qualifiée. Cela aura aussi des effets positifs sur le taux de chômage. Il s'agit donc d'un processus de restructuration économique dans lequel la création de nouveaux rapports de propriété exige la transformation des formes d'organisation. Ceci accélérera la décentralisation ou la division des anciennes entreprises nationales. 


\section{Bibliographie}

Az (1988-1990), « Conception industrielle et commerciale », l'analyse des années 1980-1990.

BARTa, Györgyi (1990), « Processus centre-périphérie dans le développement territorial économique hongrois », Pécs.

BARTA, Györgyi (1991), «Les changements dans l'organisme des sociétés industrielles et la formation de la structure industrielle dans l'espace », Budapest, p. 61-67.

IPAR (1991), « Conception de politique industrielle et commerciale », Információ.

József, Peter (1991), «Une entreprise ne peut être sauvée avec l'argent des contribuables », conversation avec le Ministre Akos Bod Péter.

KorNAI, János (1989), « Dépliant dans l'affaire de la transition économique ».

Poszmik, Erzsébet (1991), «Études de cas dans des entreprises industrielles de Budapest », Kézirat.

Poszmik, Erzsébet (1991), «Les caractéristiques des débuts de la privatisation hongroise », p. 18.

PoszMIK, Erzsébet (1991), « Les premiers signes d'adaptation économique dans la région en crise de Borsod ».

SÁrKöZY ,Tamás (1979), « La direction économique et les entreprises », Budapest.

SÁrköZY, Tamás (1989), « La préparation de la loi sur les sociétés », p. 40-60.

Schwertzer, Ivan (1982), « La taille de l'entreprise », Budapest, p. 147.

SZAB6, Györgyné (1991), « La transformation des systèmes d'organisation dans les entreprises industrielles dans le département de Borsod-Abauj-Zemplén », Miskolc.

SzallaI, Erzsébet (1990), « Pouvoir et économie».

Szallar, Erzsébet (1982), « Le processus de réforme et les grandes entreprises 》.

Szallar, Erzsébet (1988), «Les essais de réforme et les intérêts des grandes entreprises dans les années $80 »$.

SZAIlaI, Erzsébet (1991), « La métamorphose du pouvoir », p. 1-26.

VoszKa, Éva (1988), « Réforme et réorganisation dans les années 80. Questions économiques actuelles », Budapest. 